\title{
EL TRABAJO DE REFLEXIÓN EN DÍADA: LA VALORACIÓN DE ESTA EXPERIENCIA DESDE SUS ACTORES
}

\author{
Irsa Cisternas F.* \\ Carol Torres F.
}

\section{RESUMEN:}

Discursos provenientes de la esfera académica, política y de la comunidad científica en el ámbito educacional concuerdan en la importancia de relevar el desarrollo de prácticas reflexivas en la formación inicial y continúa de los profesores. En este propósito, de manera progresiva, han ido instalando aportes teóricos y metodológicos tanto en investigaciones como en dispositivos de capacitación con énfasis en estos procesos de desarrollo profesional. Este trabajo tiene como antecedente un proyecto que incorpora de manera sistemática y permanente un modelo de capacitación, con soporte en el desarrollo de procesos reflexivos por medio de un trabajo en díada. El objetivo de nuestro estudio es conocer, a través de los discursos de profesoras que participaron de esta experiencia, la valoración e impacto que tuvo en algunos de los componentes de su accionar pedagógico y profesional.

Palabras clave: prácticas reflexivas, formación de profesores, componentes de la acción pedagógica.

\section{THE WORK OF DYAD REFLECTION: THE APPRAISAL OF THIS EXPERIENCE FROM ITS STAKEHOLDERS}

\begin{abstract}
:
Speeches from the academia, politics and the scientific community in the field of education agree on the importance of relieving the development of reflective practice in initial and continuing training of teachers. In this way, in a progressive manner, have been installing both theoretical and methodological research and training devices with an emphasis on professional development of these processes. This work is based on a project that incorporates a systematic and permanent training model with support in the development process through a reflective work in Diada. The aim of our study is to know, through the discourses of teachers who participated in this experience, and impact assessment, which took in some of the components of their work teaching and professional.
\end{abstract}

Keywords: reflective practice, training of professors, components of the pedagogical action.

* Profesora de Español, Magíster en Educación, Dra. (c) en Ciencias de la Educación, Docente de la Universidad Católica de la Santísima Concepción. Email: icisternas@ucsc.cl

* Profesora de Historia y Geografía, Magíster en Educación, Dra. (c) en Ciencias de la Educación, Docente de la Universidad Cardenal Silva Henríquez. Email: cmtorreI@uc.cl 


\section{Antecedentes}

\section{I.I. Discursos y Programas oficiales}

A nivel general existe consenso en reconocer a la institución educativa como una instancia potente para transformar la educación y el compromiso para el cambio, aspectos que implicarían convertirla en una organización que desarrolla capacidades para funcionar con inteligencia y con un sentido de unión en sus propósitos y acciones (Marcelo, 1999); es decir, una institución que comparte y significa las preocupaciones educativas y contextuales en el marco de un trabajo conjunto entre sus actores.

Lo anterior encuentra respuesta a nivel de políticas educativas en el énfasis de instalar procesos de cambio a nivel de gestión de aula, formación inicial y continua de los profesores con vistas a fortalecer el desarrollo profesional docente basado en los conocimientos y la capacidad reflexiva de sus actores (Ávalos, 1994). Emerge de esta visión la idea que la mejora de la escuela estaría asociada, entre otras, al aprendizaje de los profesores construido en espacios propicios para la interacción de conocimientos, experiencias y desarrollo de procesos reflexivos (Marcelo, 200I).

A nivel de discursos oficiales, el Informe de la Comisión sobre Formación Inicial Docente (2005) incorpora dentro de sus conclusiones la necesidad de abordar la reflexión como un eje central dentro de la práctica profesional, en la medida que favorece el desarrollo de procesos reflexivos, construcción de saberes profesionales y fortalece la identidad docente: "las capacidades personales bacen referencia al sí mismo, a la construcción de la identidad profesional, al trabajo con las creencias, con la vocación, en una dinámica de reflexión permanente sobre el propio proceso de constitución como profesional de la docencia" (Informe sobre Formación Inicial Docente, 2005: 74).

En el marco de programas de formación inicial docente y de formación continua, algunas de las iniciativas que relevan las prácticas reflexivas como una herramienta efectiva para el desarrollo profesional 
las encontramos en el proyecto de Fortalecimiento de la Formación Inicial Docente (PFFID); el Marco para la Buena Enseñanza; los Grupos Profesionales de Trabajo GPT y las Redes Locales. Todas estas implementaciones, en mayor o en menor grado, tienen como uno de sus sustentos la visión de "concebir al docente como sujeto que construye conocimiento no sólo desde la apropiación crítica de lo construido por otros, sino que también a partir de la reflexión individual y colectiva acerca de su formación docente" (MINEDUC, 2002:12). Bajo esta lógica se posiciona fuertemente la idea de cambio en los procesos de formación de los profesores y la práctica reflexiva como uno de sus pilares que la favorece y potencia.

Al parecer, abundantes son las evidencias que hacen de la práctica reflexiva una estrategia importante para propiciar y generar procesos de cambios en los individuos. En el caso de los profesores, debiera ser parte del desarrollo profesional docente en tanto que fomenta la autonomía, la toma de decisiones y potencia el trabajo entre pares con vistas a mejorar las prácticas pedagógicas y el aprendizaje de los estudiantes.

\section{I.2. Discursos de la comunidad científica del ámbito educacional}

Dentro de la producción académica e investigaciones orientadas al estudio y desarrollo del pensamiento reflexivo (Contreras, I997; Ávalos, 2004; Cornejo, 2003; Cochran-Smith, 2003; Zeichneir,I993; Schön, 1992; Korthagen, 1999, 200I; Perrenoud, 200I, 2004; Tezano, 2007; Beas, 2008), existe consenso en la relevancia que adquiere favorecer prácticas reflexivas para el desarrollo profesional docente y, en forma progresiva, han ido instalando sus aportes teóricos y metodológicos en investigaciones y propuestas de capacitación a nivel de formación inicial y continua.

Sin embargo, estos propósitos no han estado exentos de tensiones que giran alrededor de sus variadas conceptualizaciones, formas e impacto en las prácticas educacionales como consecuencia de la 
pluralidad en cuanto a sus significaciones y a su impacto político y práctico (Cornejo, 2003); al respecto, variadas son las posturas y concepciones que emergen tanto de sus aspectos teóricos como de propuestas a nivel metodológico y que las hacen transitar desde racionalidades basadas en lógicas de proceso-producto hasta las que tocan aspectos políticos e ideológicos del sistema educacional (Ávalos, 1994) ${ }^{\mathrm{I}}$.

Dentro de esta pluralidad de significaciones, encontramos que diversos autores comparten supuestos que estarían en la base de pensar prácticas reflexivas en los actores del proceso educativo. Korthagen (200I) nos dice que la práctica reflexiva tiene como uno de sus propósitos hacer profesores y estudiantes más reflexivos a partir de un proceso paulatino de búsqueda de soluciones que surjan desde la experiencia, a través del apoyo de sus pares y de un proceso de supervisión que promueva la retroalimentación en función de la toma de decisiones. Se fundamenta en la idea que el profesor puede aprender a someter su propio comportamiento al análisis crítico y hacerse responsable de sus acciones para, de este modo, encontrar el gran filtro necesario para una enseñanza que trascienda el simple entrenamiento y el uso de competencias específicas de comportamiento. En este sentido, la reflexión es un proceso mental que intenta estructurar y reestructurar una experiencia, un problema o un conocimiento. Se enfatiza el rol básico de la formación de (nuevas) estructuras mentales durante el proceso de aprendizaje y que la reflexión puede tener lugar después de la acción (reflexión sobre la acción) o durante la acción (reflexión en la acción).

En esta misma línea Perrenoud (2004) concibe la práctica reflexiva como un paradigma integrador y abierto que delimita, en el terreno de la enseñanza, una problemática y un campo conceptual todavía pendiente de construir. Comparte con Donald Schön (I992) la idea de que la autonomía y responsabilidad de un profesional no se entiende sin una capacidad de reflexionar "en la acción" y "sobre

I Para profundizar en este tema se recomienda consultar Van Mannen (I977); Schön (I992); Zeichner (1993); Jay y Johnson (2002). 
la acción"; sin embargo, repara que si bien la acción, la reflexión, el aprendizaje y los conocimientos se pueden teorizar en abstracto del contenido específico de los problemas a los cuales se enfrenta es de igual forma relevante, la formación profesional desde donde se sitúan estas problemáticas: "tener en cuenta la especificidad de cada oficio y preguntarse cómo conjugar en él el paradigma reflexivo". (Perrenoud, 2004: I4) de este modo, no se podría llevar esta lógica tal como la propone Schön al terreno de la enseñanza, puesto que su relación con los conocimientos científicos como base de la acción profesional es diferente a la de otras profesiones. En este sentido, la práctica reflexiva, según Perrenoud, "supone una postura, una forma de identidad o un babitus y su realidad no se considera según el discurso o las intenciones, sino según el lugar, la naturaleza y las consecuencias de la reflexión en el ejercicio cotidiano del oficio (ibid:I3).

Si bien los aportes conceptuales anteriormente desarrollados, presentan sus particulares formas de ser asumidas, interesante resulta la propuesta de Susan Klein (2008) en la medida que incorpora un aspecto escasamente explorado en el ámbito del pensamiento y la práctica reflexiva docente, como lo es la necesidad de abordar estos procesos no sólo a partir de una lógica lineal, racional, deductiva y metacognitiva que, si bien ha potenciado en los profesores el pensamiento crítico, de igual forma ha desconocido la dimensión emocional y afectiva. Partiendo del supuesto anterior, Klein (2008) propone un "enfoque holístico" para la reflexión de profesores sobre su práctica y que tiene como componentes medulares la intuición, imaginación, contemplación y visualización; de este modo, se concibe al sujeto con una mirada integral y a la enseñanza desde una perspectiva multidimensional, con énfasis en las necesidades emocionales y espirituales de las personas.

\section{I.3. Obstaculizadores de prácticas reflexivas}

No obstante, la importancia de relevar prácticas potenciadoras de procesos reflexivos en los profesores, es posible advertir causas tan diversas como profundas que operan a la hora de instalar dispositivos 
de formación que coloquen los acentos en estos tipos de procesos. Hargreaves (2003) enfatiza en ciertas modalidades de cultura de los profesores que impactan tanto en su trabajo profesional como en los procesos de cambio dentro de la institución educativa y que actuarían como condicionantes para instalar prácticas reflexivas entre pares: El individualismo, reconocido como un trabajo aislado del docente, en gran medida obstruye la información respecto a su propio quehacer y a un trabajo cooperativo y de intercambio de experiencias entre pares. Por otra parte, la "balcanización" de la enseñanza daría lugar al trabajo de pequeños grupos en forma aislada y enfrentados al amparo de la institución educativa.

Beas (2008) complementa lo anterior, al momento de sostener que la cultura de la escuela básica y media ha potenciado un trabajo aislado entre profesores, lo cual tensiona la idea de un trabajo cooperativo y de reflexión entre pares: "Las escuelas básicas tienen una distribución profesoralumno determinada que bace que sus profesores estén aislados ya que están a cargo de un curso y deben permanecer la mayor parte del tiempo en una sola sala." (Beas, 2008:10).

Si bien los factores anteriores no son menores al momento de pensar el desarrollo de prácticas reflexivas en los profesores, quizás una de las causas que asoma con mayor recurrencia como condicionadora en la factibilidad de instalar estos procesos, la constituyen las múltiples exigencias de trabajo que debe atender el profesor, en contradicción con los tiempos disponibles, que parecieran ser los mismos. El tiempo en el trabajo de los profesores se convierte, al parecer, en la eterna limitante para desarrollar un adecuado trabajo pedagógico y más aún para concretar propuestas de innovación. En este escenario, de manera insistente, se escucha en las voces de los profesores demandas de una organización del trabajo, que considere instancias permanentes de reflexión y que otorgue tiempos reales dentro de la carga horaria para dedicarlos a la planificación, investigación pedagógica, elaboración de innovaciones y trabajo colaborativo en equipo (Colegio de Profesores, 2006). 


\section{Contextualización del estudio}

En nuestra realidad, no son pocas las investigaciones y estudios que abordan el tema de la reflexión entre profesores e implementan dispositivos para el desarrollo de prácticas reflexivas².

En este contexto, el estudio que aquí queremos compartir, tiene como antecedente una investigación realizada en el marco de un proyecto Fondecyt ${ }^{3}$. A modo general, la línea de investigación que el proyecto de referencia desarrolla, se asume desde la lógica del cambio conceptual y la reestructuración de las creencias que tienen los profesores. Uno de los criterios esenciales considerados aquí para transitar hacia la resignificación conceptual, se concreta en la implementación de un modelo de capacitación que considere dispositivos de acompañamiento para promover procesos reflexivos de manera sistemática y permanente, en el afán de profundizar y develar las inconsistencias o tensiones entre la teoría y la práctica, tanto a nivel individual como colectivo. En específico, este proyecto instala un trabajo de reflexión entre pares por medio de un trabajo en díadas, conformadas por profesores que imparten clases en diferentes niveles de escolaridad (básica y media). Esta experiencia busca, además, promover la articulación de los componentes pedagógicos y disciplinares entre los diferentes niveles de enseñanza. Las díadas se organizan a través de un proceso de acompañamiento por parte de un mediador externo que, a través del diálogo y la reflexión, facilita la incorporación de estrategias metodológicas y soportes para promover el cambio conceptual hacia prácticas más constructivas en el aula.

Dentro de las fuentes teóricas se integran los aportes provenientes de los enfoques de la psicología cognitiva y de la sociología, en específico

2 La reciente publicación del libro Prácticas Reflexivas para la Formación Profesional Docente: ¿Qué las hace eficaces? (2008), editado por Cornejo y Fuentealba, recoge interesantes estudios e investigaciones que desarrollan el tema de la reflexión a nivel de formación inicial y continua de profesores.

3 Fondecyt 10707971. Diseño, aplicación y análisis de una propuesta de intervención para elevar la calidad del aprendizaje en el aula a partir del cambio conceptual del profesor sobre sus prácticas pedagógicas constructivistas. 
los de Donald Schön en la idea de considerar la reflexión como un proceso que se lleva a cabo en y desde la acción, basado en una visión de conocimiento de la teoría y la práctica (Schön, 1992).

\section{El estudio}

Teniendo entonces como referencia la investigación anterior, el objetivo que anima nuestro estudio es conocer por medio de los discursos la valoración que realizan dos profesoras que participaron de esta capacitación de trabajo en díada con soporte en prácticas reflexivas, y su impacto en algunos de los componentes de su accionar pedagógico.

Los participantes de nuestro estudio corresponden a dos profesores de Lenguaje y Comunicación, que imparten clases en $5^{\circ}$ básico y $I^{\circ}$ de enseñanza media, en un colegio Particular Subvencionado de la Región Metropolitana.

Como estrategia de obtención de información se aplicaron entrevistas en profundidad, sin considerar un orden temático prefijado, sino que a partir de la formulación de preguntas orientadoras para acceder a la información que emerge de los discursos construidos en la práctica y experiencia directa de los protagonistas de la acción (Sandin, 2003; Rodríguez, 1999); en específico, las cuestiones que guiarán nuestra entrevista serán, de preferencia, de tipo "experiencia/conducta" (Patton, 1980 en Rodríguez 1999), en la medida que se formulan para "conocer lo que hace o ha hecho una persona" y a través de ellas se puede acceder a la descripción de experiencias, conductas, acciones" (ibid, 174).

\section{I. Análisis de la información}

Para efectos del análisis, recogemos algunos aportes provenientes del Análisis de Contenido (Klippendorf, 1990) y del Análisis de Dato Cualitativo (Rodríguez, 1999), lo anterior se justifica en la medida que, no obstante ambas formas comparten técnicas en común para abordar el tratamiento de los discursos, aquello que los hace diferentes 
es que en el caso del segundo de los procedimientos, se preserva su naturaleza textual sin recurrir a técnicas estadísticas, lo que lo aleja del análisis de contenido (Rodríguez, 1999), criterio que en este estudio se considera.

Nuestro procedimiento de análisis contempla, para la reducción de datos el "criterio teórico" y para el proceso de "síntesis y agrupamiento" (Rodríguez, I999), que nos centraremos en el estudio de la Progresión Temática, en específico los conceptos de Tema y Rema (Escuela de Praga), con el propósito de identificar con ello la progresión temática que se va desprendiendo de los tópicos (Atienza, 1994).

A modo de aclaración conceptual, entenderemos por Tema "aquello que contiene lo ya conocido o presupuesto y que, en consecuencia, posee la menor información en un contexto dado o en una situación de enunciación." (Bernardez, I982: 26) y por Rema "lo que aporta el contenido fundamental del mensaje en un contexto dado o en una situación determinada, lo que expresa lo nuevo, lo que se comunica acerca del tema." (ibid, I27). La articulación coherente de información conocida con información nueva en el discurso es lo que llamaremos "progresión temática".

Los Temas aquí considerados en los discursos de las profesoras son:

- Experiencia de trabajo en díadas

- Trabajo de planificación

- Elaboración de materiales

- Evaluación de aprendizajes

- Aprendizaje personal

- Impacto en el trabajo docente

- Trabajo en díadas como cambio en el trabajo profesional

- Trabajo en díada como propuesta factible a futuro.

Una vez identificados los Temas orientadores de los discursos, se articuló esta información con los respectivos remas (R); es decir, con 
la información nueva que va nutriendo los respectivos temas y que nos permitirán a través de su progresión temática, ir develando la valoración e impacto que tuvo el trabajo en díadas en estas profesoras. Por último, es necesario advertir que para el análisis consideramos además, subremas ( $\mathrm{r}$ ) en tanto nueva información aportada al rema principal (R).

\section{Análisis de la Progresión Temática en los Discursos}

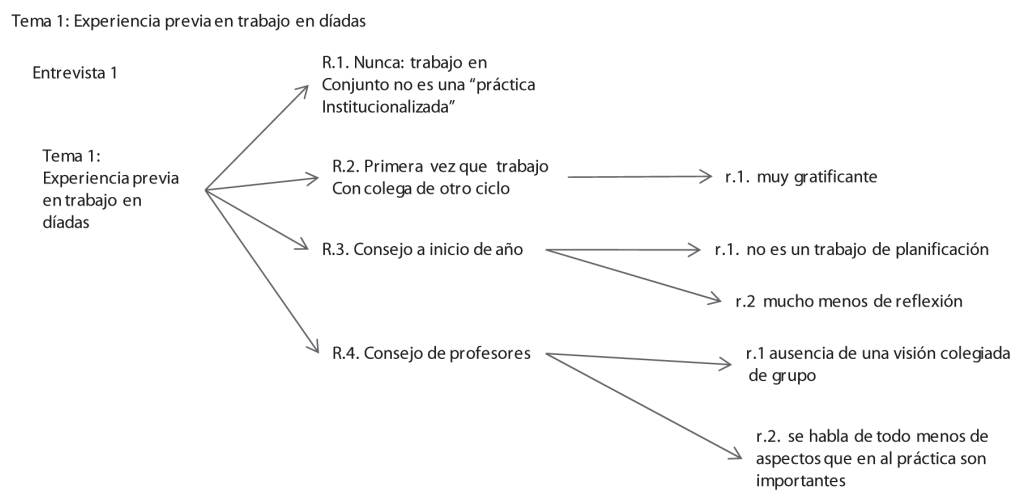

Entrevista 2

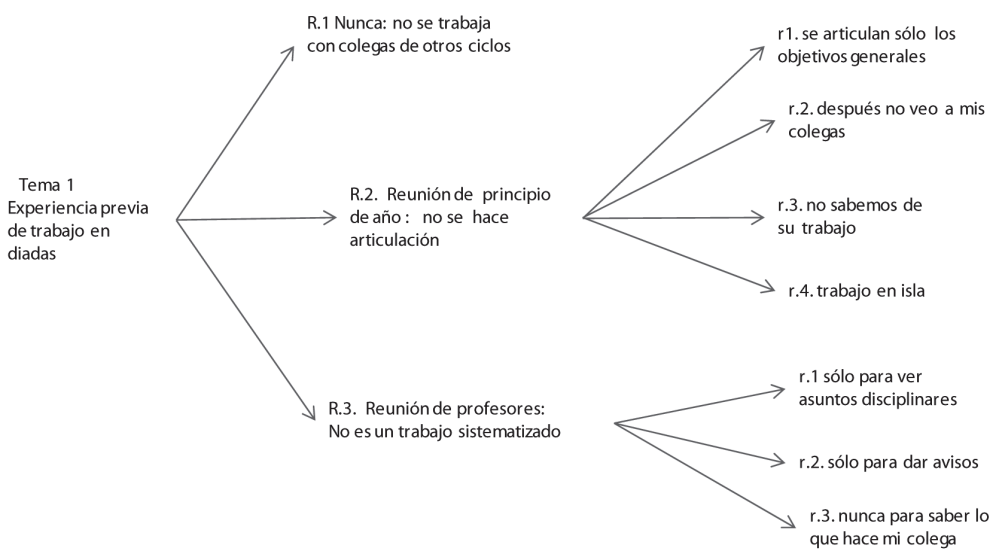

Se desprende de la información, la ausencia en el trabajo profesional de prácticas que considere el trabajo en díadas; al parecer, momentos determinantes para tomar decisiones a nivel de gestión y del trabajo 
pedagógico, como lo son los consejos de inicio de año y las reuniones, según lo declarado, no son instancias sensibles al tratamiento de estos temas ni mucho menos al desarrollo de prácticas basadas en el diálogo, la reflexión, el trabajo colaborativo y de intercambio de experiencias entre los profesores, más bien las relaciones al interior de este espacio escolar operan desde lógicas que fortalecen el aislamiento e individualismo entre pares y, en consecuencia, impactan el trabajo pedagógico.

TEMA 2: La planificación en el trabajo en diadas

Entrevista 1

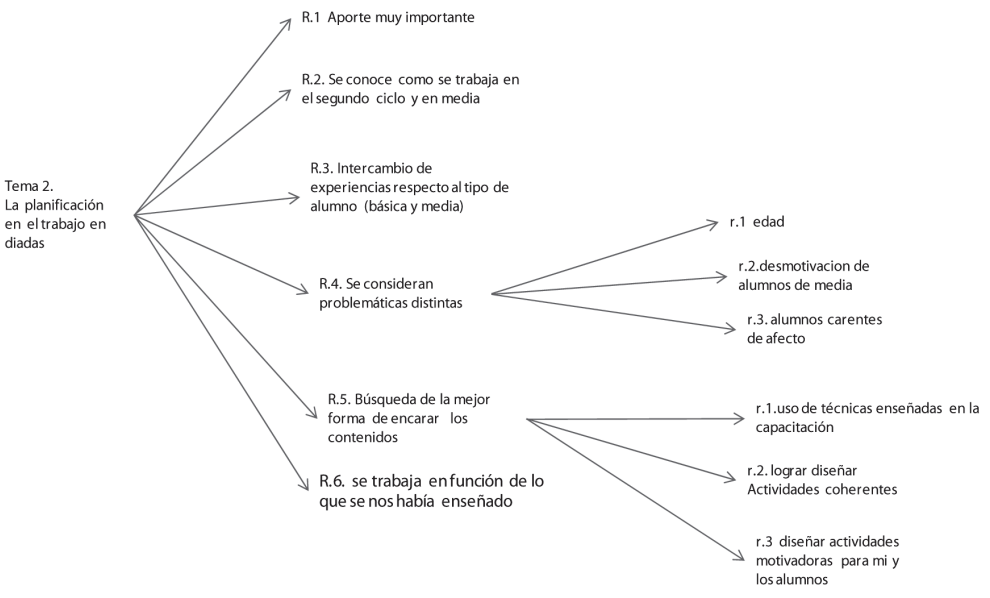

Entrevista 2

Tema 2:

La planificación en el trabajo en diadas

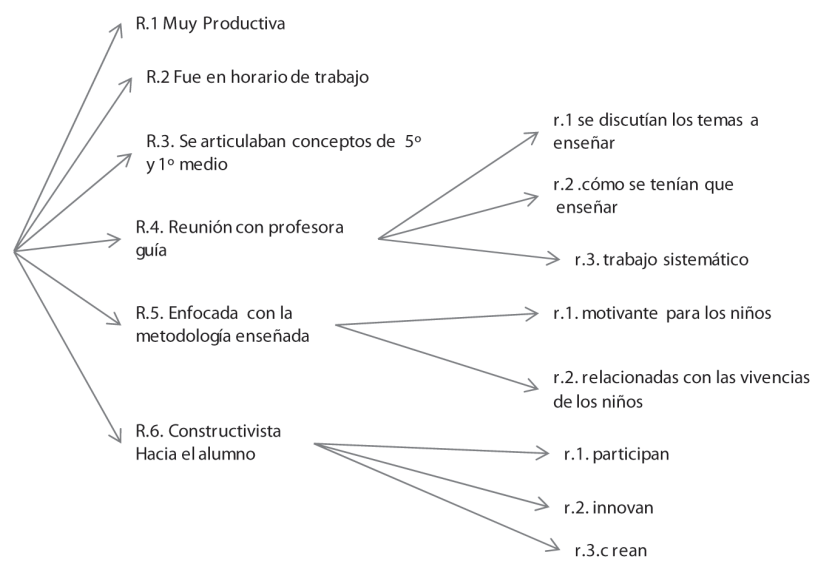


La posibilidad de trabajar una experiencia que considere el trabajo en díadas, impacta favorablemente los procesos de planificación del trabajo pedagógico. Los componentes y procesos que incorpora esta nueva forma de trabajo, se instalan desde una perspectiva que considera elementos anteriormente ausentes en las prácticas pedagógicas de los profesores.

Los dispositivos que van dando cuerpo a la planificación, se abordan desde una mirada contextualizada en atención a las diversas "problemáticas" del sujeto al cual se enseña.

Se valora la posibilidad de poder realizar la labor de planificación durante la jornada de trabajo y asistido por una profesora guía, estos dos factores incentivaron a la reflexión y discusión entre mediador y pares; al parecer, es en esta dinámica donde la figura del "acompañante" se asoma como un "experto", que orienta lógicas de acción en atención a la articulación por niveles de enseñanza, la pertinencia de los contenidos y de los aspectos técnicos de la planificación, entre otros.

TEMA 3. Elaboración de materiales en el trabajo en diadas

Entrevista 1

Tema 3: Elaboración de materiales

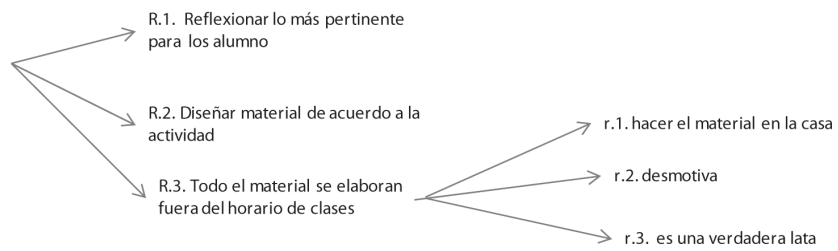

Entrevista 2
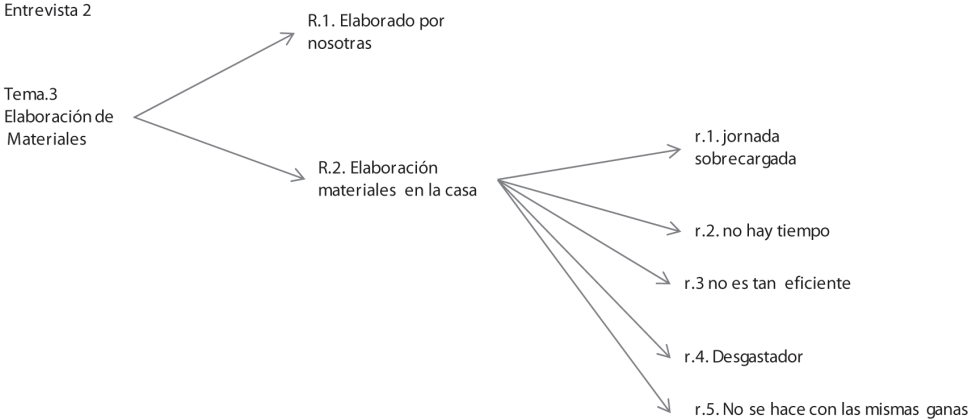
En el tema referido a la planificación, uno de los aspectos que se valoran favorablemente es el poder desarrollar esta actividad dentro de la jornada de trabajo; sin embargo, no contar con estos mismos tiempos para la elaboración de materiales y, en consecuencia, destinar para ellos otros tiempos, hace que esta actividad se convierta en una instancia que desmotiva y que no se asuma con el mismo entusiasmo y motivación que el haberla realizado dentro de la jornada laboral.

Tema 4. La evaluación de los aprendizajes en el trabajo en díadas
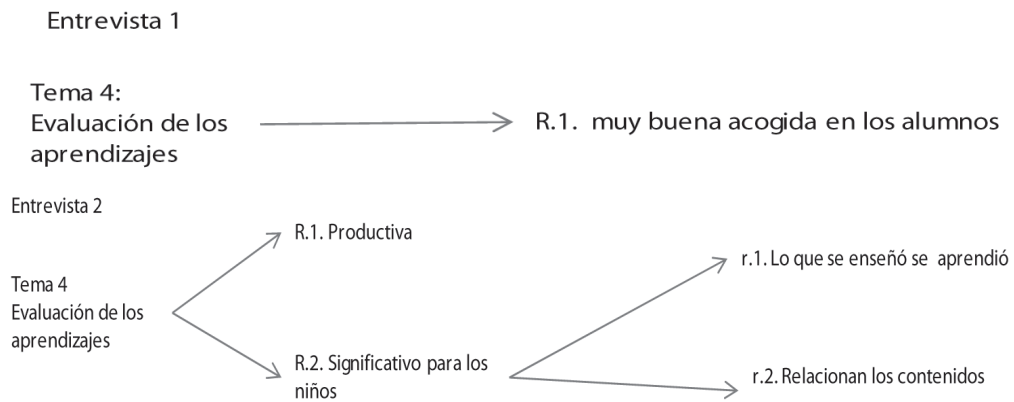

Las formas como se describe la evaluación la sitúan desde una visión constructivista, en la medida que los estudiantes logran un aprendizaje significativo, que permite relacionar y contextualizar los contenidos; lo cual supone que los dispositivos elaborados para evidenciar los aprendizajes de los estudiantes se abordan en coherencia con la planificación y elaboración de materiales; es decir, existiría una correspondencia en el qué se enseña, cómo se enseña y cómo se evalúa.

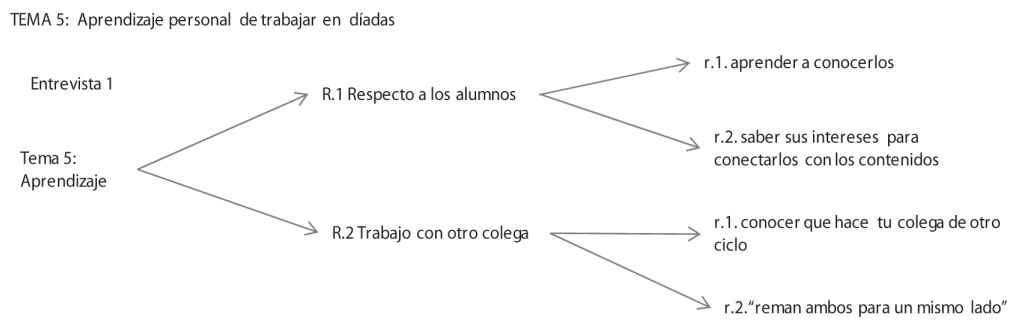


El trabajo de reflexión en díada: la valoración de esta experiencia desde sus actores - Irsa Cisternas F. y Carol Torres F.

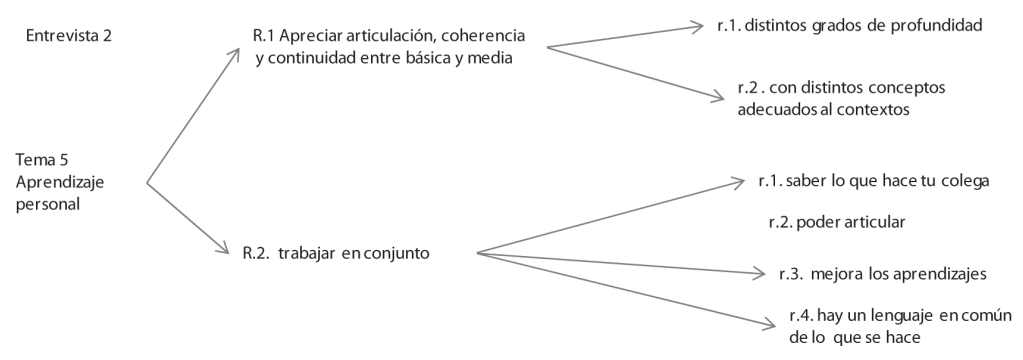

En cuanto al aprendizaje personal que recoge de haber participado en esta experiencia, destaca el relevar la importancia de conocer quién es el alumno al cual se enseña y conectar los intereses de éstos con las materias a enseñar; articular con profesores de básica y media en un trabajo conjunto de construcción de aprendizaje, y la posibilidad de aprender y compartir experiencias con un par. El aprendizaje personal se vio reflejado en la idea de aprender a trabajar en conjunto, principalmente en función de conocer y retroalimentar sus propias experiencias.

Tema: 6 Impacto en el trabajo profesional

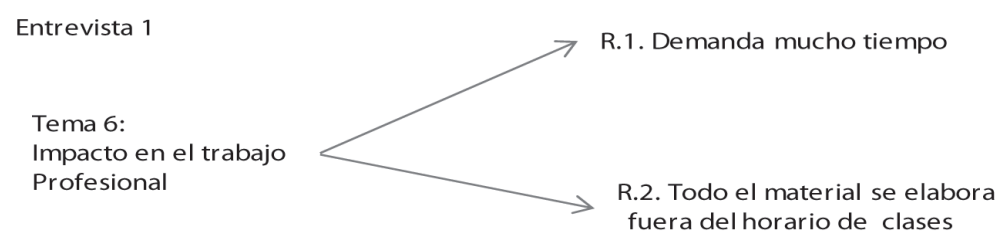

Entrevista 2

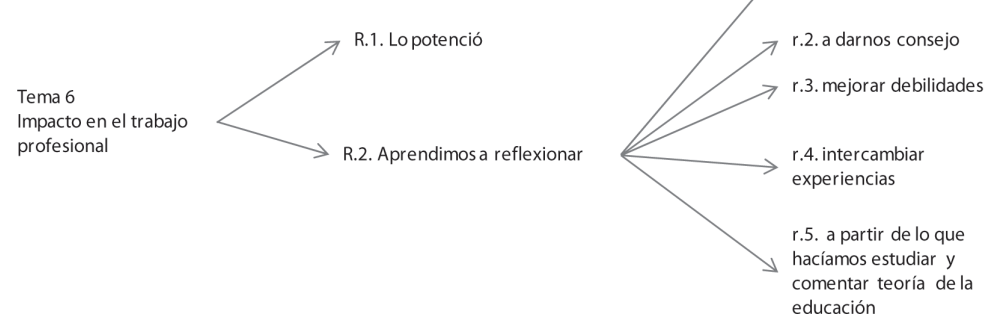

Respecto a este tema, hay tensiones en cuanto a los discursos declarados: por una parte, el segundo de los discursos declara una valoración positiva de esta experiencia y en el impacto del trabajo profesional en la medida que se aprendió a reflexionar y, con ello, 
acceder a prácticas altamente significativas de intercambio, análisis y profundización y discusión teórica; sin embargo, para la otra profesora, el factor tiempo se constituye en una condicionante negativa respecto al impacto en el trabajo profesional.

TEMA 7: Trabajo en diada como cambio en el trabajo profesional

Entrevista 1

Tema 7:

Trabajo en díada como cambio
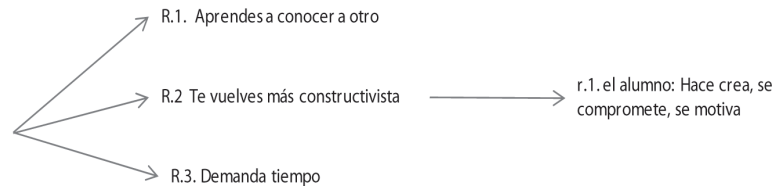

El cambio se concibe en la idea de convertirse más constructivista, lo cual permitiría plasmar procesos en concordancia con esta visión más integral del alumno; sin embargo, nuevamente aparece el factor tiempo como una condicionante obstaculizadora para el desarrollo de prácticas constructivistas.

TEMA 8: Trabajo en diada como propuesta factible a futuro

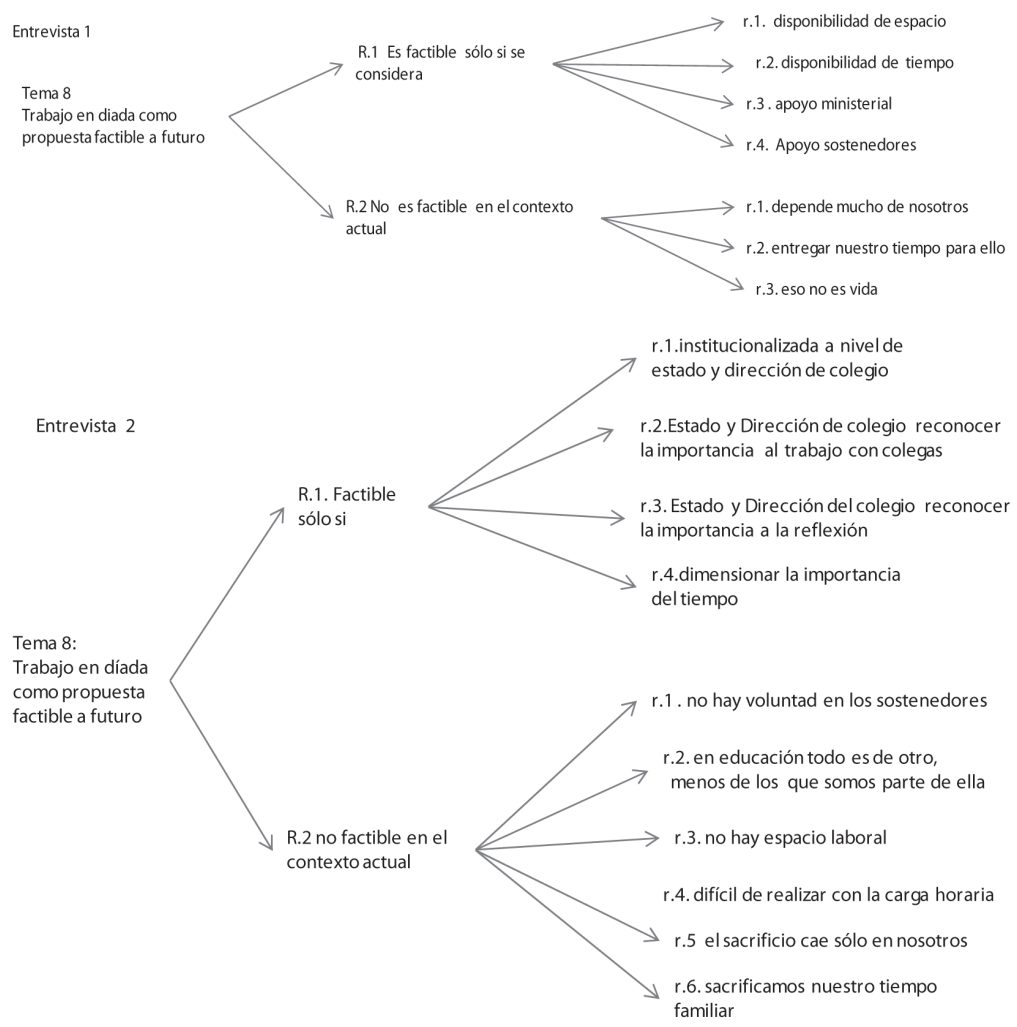


Surgen tensiones al momento de abordar la factibilidad de este trabajo en díadas en el sistema educativo. En lo concreto, dadas las condiciones ofrecidas tanto a nivel de políticas educativas como a nivel directivo institucional, resulta imposible en el actual contexto instalar propuestas de innovación con las características que demanda este proyecto. Sólo es factible concretar prácticas de cambio sostenido en el tiempo, si se releva la importancia que tienen los propios actores dentro del proceso educativo, considerando sus lógicas de funcionamiento, espacios propicios (tiempo, carga horaria) para una adecuada implementación.

\section{Conclusiones}

El análisis de los discursos de las profesoras, respecto a la valoración e impacto que tuvo en algunos de los componentes de su trabajo pedagógico el haber participado de esta experiencia, nos permite observar que:

Con relación al aprendizaje personal que esta experiencia rescata, encontramos que la coordinación y articulación para el tratamiento de contenidos y su enseñanza entre los niveles de básica y media constituye un aspecto novedoso, relevante y altamente significativo para las profesoras, que permite abrir espacios para el intercambio de experiencias, interacción mutua y comprensión compartida respecto a una puesta en común: "remar ambos para un mismo lado" (entrevista I).

Por su parte, en el tema "el trabajo en díada como cambio en el trabajo profesional" se reitera un componente ya mencionado en tema anterior, como lo es la posibilidad de potenciar el diálogo académico entre pares, profundizar en el manejo conceptual, lo cual obliga, de acuerdo a lo declarado, a estar más preparadas teóricamente y a impulsar un ejercicio permanente de actualización disciplinar y teórica.

Se reconoce a través de los discursos que, en general, los marcos explicativos para el análisis, la reflexión y el trabajo pedagógico 
se asumen desde una mirada constructivista, en la medida que la planificación de actividades y los dispositivos de aprendizaje se construyen considerando los intereses de los estudiantes y una participación activa y creativa de éstos, componentes que, sin duda, estimulan el acercamiento significativo y útil de conocimiento. Lo anterior, en alguna medida, encuentra sentido con lo señalado por Wertsch (1999) cuando enfatiza en la necesidad de propiciar la modificación de las estructuras de participación en la enseñanza, principalmente, en la relación profesor/ alumno y a una participación activa de estos últimos.

Como se mencionó en su momento, el proyecto de referencia declara, entre otros, adscribirse a un enfoque de corte más sociológico de la práctica reflexiva; en específico, a partir de la propuesta presentada por Schön (1992). Al respecto, se puede advertir que la forma como es asumido el trabajo de planificación se acercaría a una racionalidad de tipo práctica en la medida que se potencia el diálogo, la reflexión y discusión en la "búsqueda de la mejor forma de encarar los contenidos" (entrevista I), además del rol del mediador asumido como un práctico experimentado que acompaña, orienta e induce la reflexión y discusión de las formas y temas a enseñar; sin embargo, estimamos que a la hora de explicitarse en estos discursos las estrategias a utilizar, la idea anterior pierde fuerza cuando se declara que el trabajo de planificación se realiza "en función de lo que se nos babía enseñado" (entrevista I), lo cual sitúa al mediador desde una posición de experto y a los profesores más bien como reproductores de estas estrategia.

En este sentido, no se advierte con claridad la presencia de uno de los factores que condicionan una racionalidad de tipo práctica en este proceso, como lo es la presencia de un tipo reflexión que involucre la reconstrucción de la experiencia y la capacidad de aprender de las situaciones prácticas de modo problematizador (Schön, I992). Si bien, el proyecto recoge y desarrolla algunos componentes aportados desde esta racionalidad, la implementación de esta propuesta, dadas las formas como se configura la relación acompañante/díada se asocia, de preferencia, a la lógica de acción postulada por D. Cruikshank (1987) y su modelo de "práctica reflexiva”, dado que la reflexión es 
vista como un soporte instrumental, "basada en el logro de los objetivos y la entrega de información para pulir sus destrezas en desarrollo" (Cornejo, 2003:349), cuyo propósito final sería potenciar habilidades para analizar las prácticas docentes. En este sentido, el nivel de reflexión que alcanza correspondería al técnico (Van Mannen, 1977), con énfasis en la reflexión acerca de la efectividad de las estrategias que implementan en el aula las profesoras.

Con relación a los factores que restringirían la implementación de esta experiencia, "el trabajo de elaboración de materiales" se convierte en una actividad que afecta de manera desfavorable el trabajo de las profesoras. Si bien, la forma como se asume este componente encuentra correspondencia con uno de los fundamentos de esta propuesta como lo es el incentivar un trabajo reflexivo con vistas al desarrollo de prácticas constructivistas, lo anterior pierde fuerza al asociarlo con un factor determinante como lo es la sobrecarga horaria de las profesoras. El no disponer de los tiempos necesarios para el desarrollo de esta actividad, obliga a ocupar otros tiempos: "los destinados al espacio familiar" y que, en definitiva, hace que un recurso tan esencial dentro del trabajo pedagógico que articula y concreta en el aula los dispositivos de la planificación se convierta en una práctica poco eficiente que desmotiva y desgasta.

Al parecer, el escaso tiempo del que disponen las profesores para implementar y desarrollar procesos de innovación, se constituye en un factor determinante que obstaculiza su factibilidad. La falta de consideración a nivel de políticas ministeriales y de los directivos del establecimiento, respecto a la sentida necesidad de disponer del tiempo necesario para un adecuado trabajo pedagógico, nos hace suponer que se está frente a dos dimensiones de tiempo que tensionan y separan los intereses, responsabilidades y perspectivas entre los directivos y profesores. Al respecto, Hargreaves (2003) sostiene que en la medida que los directivos están más alejados del trabajo en aula, no logran dimensionar las complejidades de los procesos que a su interior ocurren. Esta visión monocrónica, hace que los directivos perciban que las implementaciones y procesos del aula se desarrollen con lentitud; contrario a esta visión, e inmersos en las presiones y 
complejidades propias del espacio pedagógico, los profesores perciben que el tiempo transita en forma rápida con relación a las múltiples demandas que hay que atender.

Dentro de esta misma línea, se podría pensar, además, que uno de los factores obstaculizadores para que este tipo de implementación quede instalada a futuro podría obedecer a la naturaleza de este cambio (Fullan, 2002). En este sentido, nos encontraríamos frente a una problemática, respecto al modo como se introduce el cambio, con relación a las bajas oportunidades reales que tiene la institución educativa para favorecer procesos de reflexión y aprendizaje sostenido por parte de los profesores. Quizás si las propuestas de cambio surgieran como una necesidad compartida por todos los integrantes de esta comunidad escolar, sería más probable que las condiciones sociales del cambio influirían de manera favorable en la asignación de los espacios y tiempos adecuados para la implementación de esta propuesta de innovación. En definitiva, estimamos que de no considerarse variables como la excesiva carga horaria y, en consecuencia, la escasez de tiempo de que disponen las profesoras, lamentablemente, se complejiza la posibilidad de instalar en su trabajo pedagógico sostenido, el desarrollo de los componentes.

Por otra parte, las profesoras comparten que anterior a esta experiencia, el trabajo de reflexión en díadas no es una práctica considerada dentro de su espacio de trabajo; es más, las características que reflejan a esta institución educativa están asociadas a relaciones que favorecen un trabajo aislado, desarticulado y ausente de reflexión académica. En este sentido, la cultura escolar que aquí se constituye, no obedece a una decisión personal o a un tipo de individualismo electivo (Hargreaves, 2003) sino, más bien, es fruto de las condicionantes administrativas, directivas y horarias que impedirían un trabajo colaborativo, de intercambio de experiencia e información entre los profesores. Sin embargo, estimamos que si bien atender a los factores anteriores no es la intención del proyecto de referencia, puesto que está dirigido y parcializado hacia pequeños grupos disciplinares conformados por díadas que favorecen modos propios de pensar y enseñar; no obstante lo anterior, estimamos que esta implementación, 
en alguna medida, no aminora la cultura balcanizada de enseñanza ya instalada en este contexto educativo; dado que no cruza al resto de la comunidad docente, lo que, a nuestro juicio, potenciaría una baja permeabilidad respecto a los demás miembros del colegio, no participantes del proyecto.

Finalmente, podríamos concluir que muchos son los beneficios que trae, para las profesoras haber participado de un proyecto que potencie prácticas reflexivas por medio de un trabajo en díada; en el caso de esta experiencia, favorece la articulación tanto disciplinar como de nivel de enseñanza, vincula y genera nuevas estrategias de apoyo en beneficio de los procesos de enseñanza y aprendizaje; motiva a la reflexión y al diálogo académico; posibilita compartir experiencias y avanzar hacia prácticas más constructivistas en el trabajo pedagógico; lamentablemente, todas estas fortalezas chocan con una cultura tradicional y rígida que privilegia el individualismo, la balcanización y prácticas lejanas al diálogo académico y reflexivo. En este sentido, estimamos que se tensiona, desde la lógica que el proyecto demanda, la posibilidad de pensarla como una práctica factible de quedar instalada en el tiempo.

\section{Bibliografía}

Atienza, E. (1994). "La progresión temática en el discurso académico". Actas del XII Congreso Nacional de la Asociación Española de Lingüística Aplicada AESLA. Nuevos Horizontes de la Lingüística Aplicada. Barcelona (pp. $33-40)$.

Ávalos, B. (1994). "Creatividad versus autonomía profesional del profesor: Consideraciones sobre el tema, derivadas de la investigación pedagógica”. Revista Pensamiento Educativo, Nº I4 (pp. I4 - 47).

Bernárdez, Enrique (1982). Introducción a la Lingüística del Texto, Espasa-Calpe, Madrid.

Beas, J.; Gómez, V.; Thomsen, P. (2008). “CCómo cambian los profesores con la práctica reflexiva?”. En Prácticas reflexivas para la formación inicial docente: ¿qué las bace eficaces? Ediciones Universidad Católica Silva Henríquez, Santiago (pp. II3 - I40). 
Cornejo, J. (2003). "El pensamiento reflexivo entre profesores". Revista Pensamiento Educativo $\mathrm{N}^{\circ} 32$ (pp. 343 - 373).

Colegio de Profesores (2006). "La Crisis del sistema educativo".

Fullan, M. (2002). Los nuevos significados del cambio en la educación. Ed. Octaedro, Barcelona.

Hargreaves, A. (2003). Profesorado, cultura y posmodernidad (cambian los tiempos, cambia el profesorado). Morata, Madrid.

Informe de la Comisión sobre Formación Inicial Docente (2005)

Klein, S. (2008). "Holistic reflection in teacher education: issues and strategies". Reflexive practice N. 9 (pp. III - I2I).

Kothagen \& Kessels (1999). "Linking Theory and Practice: Changing the Pedagogy of Teacher Education”. Educational Researcher, 28 (pp. 4 - I7).

Korthagen, F. (200I). "Working with groups of student teachers". En F. Korthegen, Linking practice and theory: the pedagogy of realistic teacher education, (pp. 149 - 174). L. Erlbaum Associates, Mahwah, N.J.

Marcelo, C. (1999). "Cultura escolar y cultura profesional: los dilemas del cambio". Revista Educar N ${ }^{\circ} 24$. (pp. 47 - 69).

Marcelo, C. (200I). La construcción del conocimiento en la enseñanza. Síntesis, Madrid.

Ministerio de Educación (2005). Informe Comisión formación inicial docente. Santiago, Chile.

Ministerio de Educación (2002). "Desarrollo profesional docente (experiencias de colaboración en enseñanza media)”. Programa de Mejoramiento para Enseñanza Media.

Perrenoud, Ph. (2004). Desarrollo de la práctica reflexiva en el oficio de enseñar. Profesionalización y razón pedagógica, Graó.

Rodríguez, G.; Gil, J. \& García, E. (I999). Metodología de la investigación cualitativa. Aljibe, Málaga.

Sandín, E. (2003). Investigación cualitativa en educación. Fundamentos y tradiciones. Mc Graw and Hill, Madrid.

Schön, D. (1992). La formación de profesionales reflexivos: bacia un Nuevo diseño de la enseñanza y el aprendizaje en las profesiones. Paidós, Barcelona.

Van Mannen, M. (1977). "Linking ways of knowing with ways of being práctical”. Curriculum Inquiry, No 6 (pp. 205 - 228).

Wertsch, J. (1999). "La acción mediada en el espacio social”. En La mente en acción (pp. 173-217). Aique, Buenos Aires. 